\title{
Evaluation of Integrated WiMAX-WLAN under Pervasive Environment in OPNET
}

\author{
Sandeepika \\ Department of Computer \\ Science \& Engineering \\ CTIEMT, Jalandhar
}

\author{
H. P. Singh \\ Department of Electronics \& \\ Communication Engineering \\ CTIEMT, Jalandhar
}

\author{
Manju Bala \\ Department of Computer \\ Science \& Engineering \\ CTIEMT, Jalandhar
}

\begin{abstract}
The integrated network provides the diverse application to the user. WiMAX is the most promising high speed broadband access with full QoS and WLAN provides high data rate at low cost, for limited coverage area. Integrated WiMAXWLAN network can be capable of providing ubiquitous connectivity and high data rate to the end user. This paper focuses on the comparative performance analyzing of integrated WiMAX and WLAN Network. The proposed architecture analyzes the performance of integrated WiMAX and WLAN Network with outdoor to indoor and pedestrian path loss model for rural environment under varying network conditions.
\end{abstract}

\section{Keywords}

WiMAX-WLAN Integration, Path Loss, Delay, Throughput

\section{INTRODUCTION}

The demand for wireless communication is increasing day by day. Since it ranges from pager system to mobile communication and include various access technologies. WLAN operates on the unlicensed $2.4 \mathrm{GHz}$ and $5 \mathrm{GHz}$ frequency band under various network conditions such as and supports data rate up to 54Mbps. The coverage area of WLAN is limited due to signal weakness at the edge of the cell and bandwidth of WLAN is also limited [2]. WiMAX, based on IEEE 802.16 standards, a broadband wireless access technology which provides high throughput, great coverage, flexible Quality of Service (QoS)support and extensive security and provides an alternative to cable and Digital Subscriber Line (DSL) [3]. WiMAX provides the fixed and mobile connectivity for point to multipoint architecture with full QoS support. Fixed WiMAX system uses an air interface based on Orthogonal Frequency Division Multiplexing (OFDM) and a robust mechanism against multi-path propagation and frequency selective fading and using adaptive modulation technique to enhance performance of network. The transmission scheme OFDM provides multimedia communications, high speed data and video. WiMAX can be serving as the backhaul connection to the WLAN hotspots and WiMAX could serve as a faster and cheaper alternative to wired backhaul for these hotspots.

Konal et al [4] analyzed the network coverage for WLAN and proposed an ordinary kriging approach to measure the signal strength of WLAN \& to reduce the cost of time consuming and labour intensive site surveys. Shrestha et al [5] analyzed the performance of mobile WiMAX environment for $2.5 \mathrm{GHz}$ and $3.65 \mathrm{GHz}$ frequency spectrum under different network conditions and proposed that $3.65 \mathrm{GHz}$ frequency spectrum is a favourable option to wireless application. Ain et al in [6] evaluated the performance of Walfisch- Bartoni path loss model in terms of Cell radius, base antenna height and frequency. Zaballos et al [7] analyzed the performance of various propagation models for wireless networks in indoor and outdoor environments and compared the performance of outdoor model with different indoor models such as linear, one slope, modified free space, Motley-Keenan and COST multi-wall. Mardeni et al [8] evaluated the performance of WiMAX environment in sub urban and open urban area under optimized COST-231 path loss model and compared the performance of optimized COST-231 Hata model with Stanford University Interim (SUI) model and the Egli model. Priyanka et al [9] analyzed the performance of different path loss model such as free space, outdoor to indoor and pedestrian environment and vehicular model and suburban under WiMAX environment for voice application. Lin et al [10] proposed a unified connection oriented architecture for the integration of WiMAX and WLAN in broadband wireless networks. WiMAX/WLAN access point used to combine the WiMAX and WLAN access technologies. The integrated architecture provided the QoS support between WiMAX and WLAN interfaces and reduces the end-to-end delays of high priority traffic. The work in this paper presents the performance evaluation of integrated WiMAX and WLAN for mobile and fixed nodes under the outdoor to indoor and pedestrian path loss model under varying network conditions.

\section{INTEGRATION ARCHITECTURE}

Integration is the process of combining two different networks for full QoS support. Integrated architecture provides benefit to both end users and service providers. The integration of WLAN and WiMAX network increases the coverage area and bandwidth. In the same manner, WiMAX can be used to connect Wi-Fi hotspot [11]. The integration of WIMAX and WLAN is shown in Fig. 1.

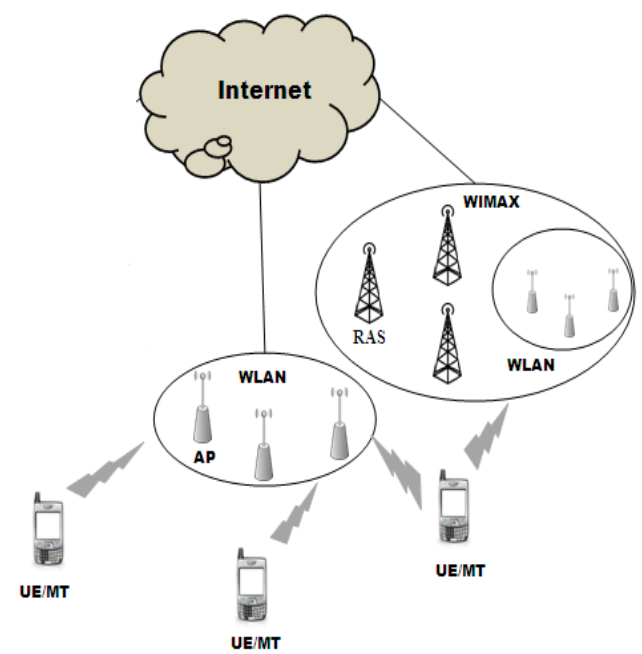

Fig. 1: Integration of WiMAX and WLAN network 


\section{1}

Method of Integration

The integrated architecture has gaining more popularity because the integrated architecture provides the endless QoS support. There are three different method of integration [12].

A) Tight Coupling: Tightly coupled architecture provides a single core network. Tight coupling reduces the handoff and provides the mobility management. But it is more complex than other coupled architecture.

B) Loose coupling: In loose coupling integration, two different networks are connected independently with the internet backbone through gateway router. Loose coupled architecture allows independent deployment and operations of the network.

C) Open coupling: In open coupling scheme, dissimilar networks are working independently and use separate authentication mechanism. The open coupling can be used for billing system.

\section{SIMULATED WIMAX-WLAN NETWORK}

The WiMAX and WLAN integrated network under the effect of outdoor to indoor and pedestrian environment path loss model has been designed and simulated in OPNET Modeler 14.5. The design parameters for integration of WiMAX and WLAN are listed in Table 1. Both the networks are integrated through the loose coupling scheme and the traffic has been generated through Weibull function. The network has been designed for the rural areas and the performance of the integrated network has been analyzed for Outdoor to Indoor \& Pedestrian path loss model. The simulation scenarios for integrated WiMAX and WLAN for fixed and mobile nodes have been presented in Fig. 2 and Fig. 3.

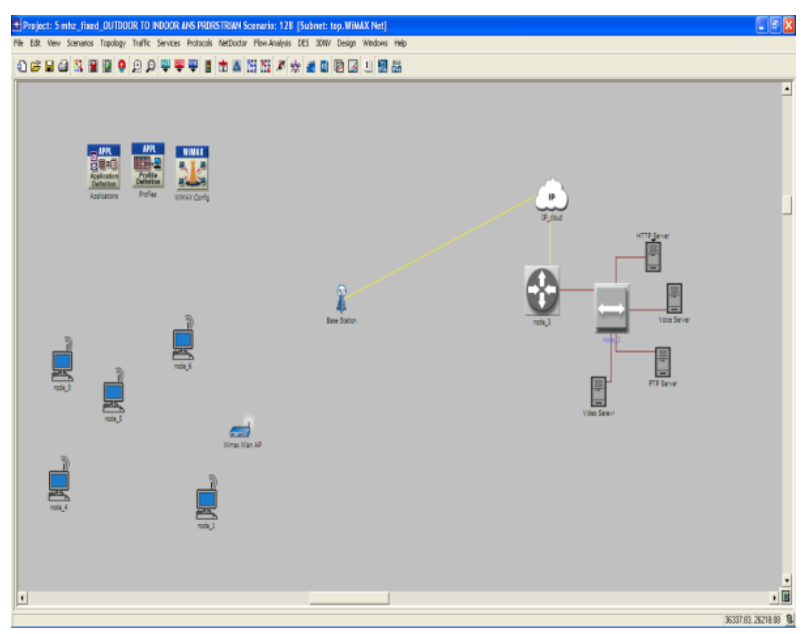

Fig. 2: Integrated WiMAX - WLAN network for fixed Nodes

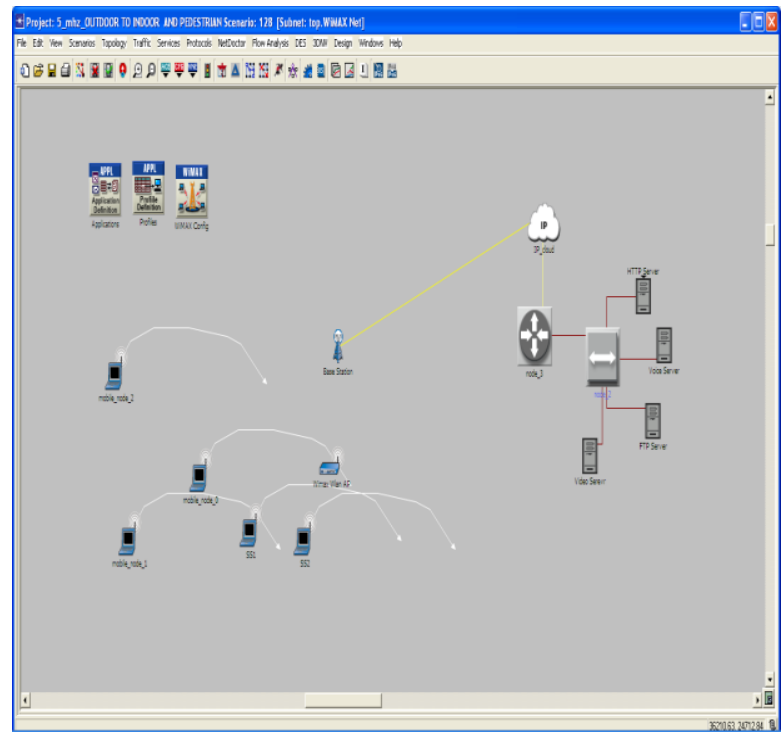

Fig. 3: Integrated WiMAX-WLAN network for Mobile Nodes

Table 1 Design parameters for integrated WiMAX-WLAN network

\begin{tabular}{|c|c|c|}
\hline Parameters & \multicolumn{2}{|c|}{ WiMAX-WLAN Network } \\
\hline $\begin{array}{c}\text { Integration } \\
\text { Scheme }\end{array}$ & $\begin{array}{c}\text { Loose } \\
\text { Coupled }\end{array}$ & $\begin{array}{c}\text { Loose } \\
\text { Coupled }\end{array}$ \\
\hline Node Type & Mobile & Fixed \\
\hline Environment & Rural area & Rural area \\
\hline Traffic & Weibull & Weibull \\
Class & & \\
\hline
\end{tabular}

\section{RESULT}

The performance of the WiMAX-WLAN integrated network has been analyzed for pervasive environment under varying network conditions such as mobility \& path loss. The integrated network has been designed in two different modes for fixed and mobile nodes for proper function in the rural areas. The performance of the fixed and mobile integrated networks has been analyzed in terms of delay, data dropped and throughput. The simulation results for the fixed integrated WiMAX-WLAN design are presented in Fig. 4 to Fig. 8. The results in Fig. 9 to Fig. 13 present the performance the mobile integrated network. 


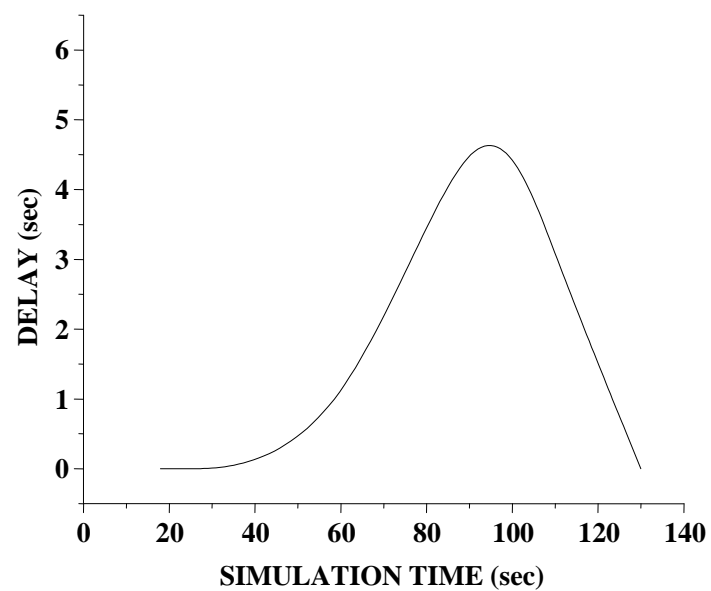

Fig. 4: Delay in WiMAX for fixed node in integrated WiMAX/WLAN network

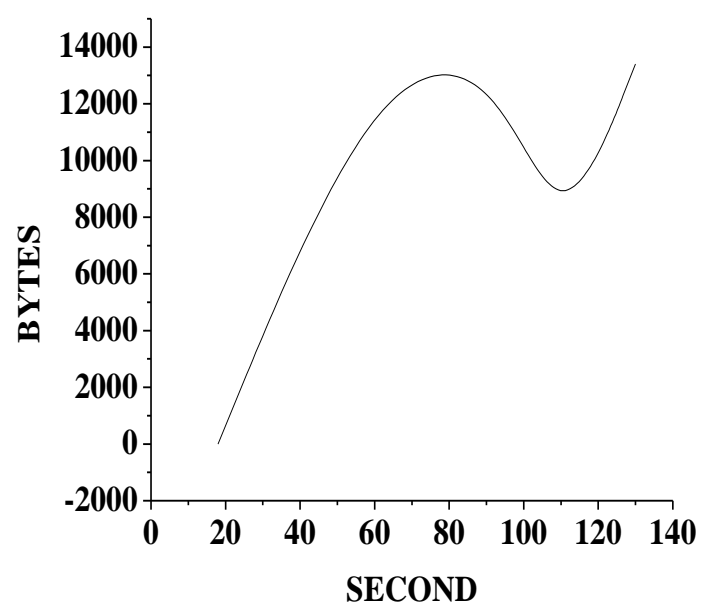

Fig. 5: Throughput in WiMAX for fixed node in integrated WiMAX -WLAN network

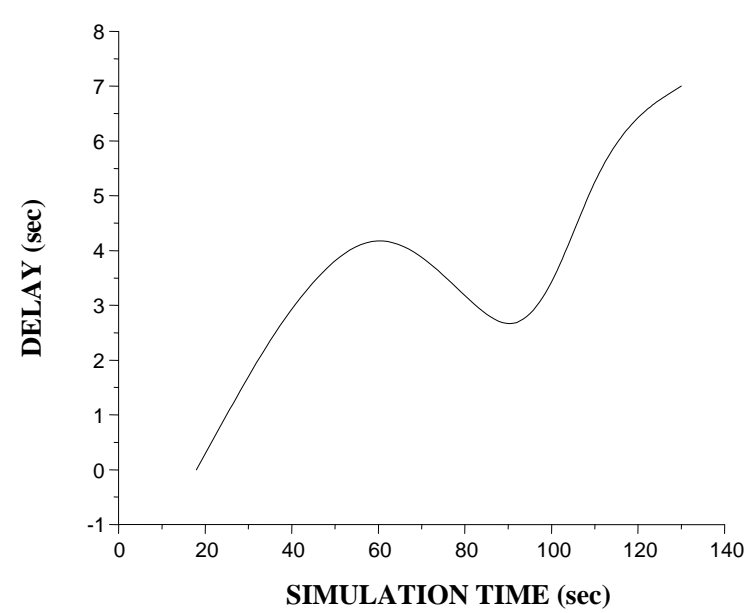

Fig. 6: Delay in WLAN for fixed node in integrated WIMAX-WLAN network

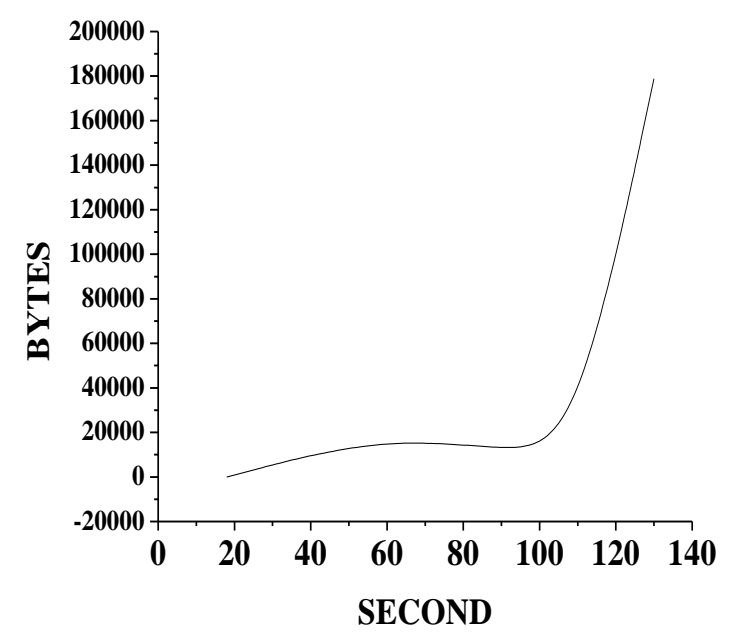

Fig. 7: Throughput in WLAN for fixed node in integrated WiMAX -WLAN network

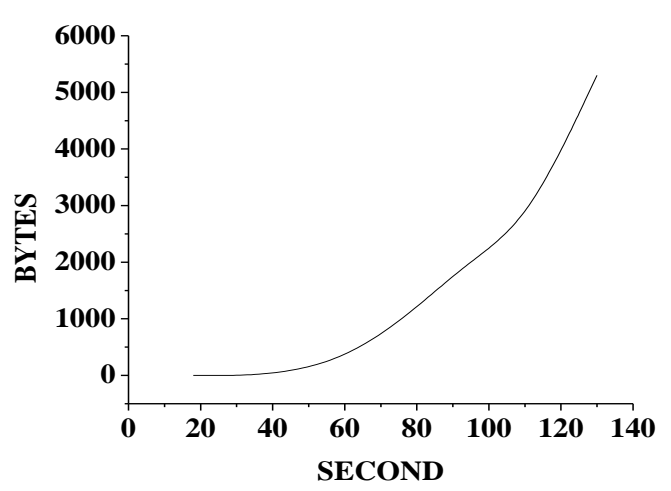

Fig. 8: Data dropped in WLAN for fixed node in integrated WiMAX -WLAN network 


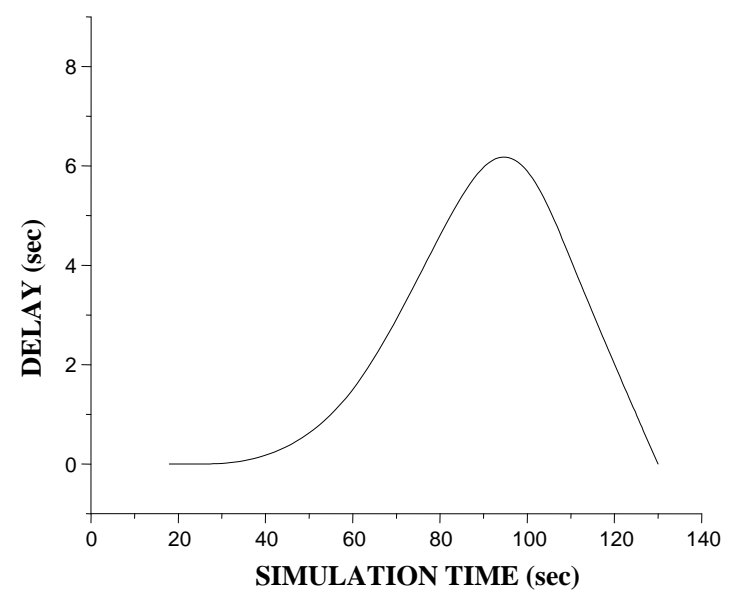

Fig. 9: Delay in WiMAX for mobile node in integrated WiMAX -WLAN network

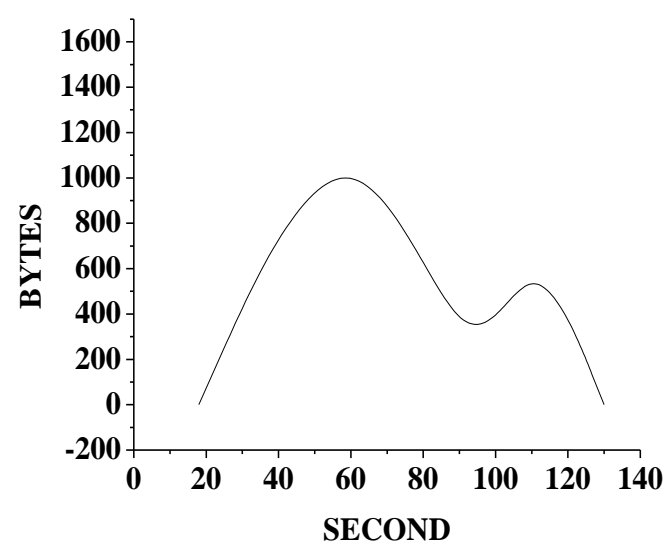

Fig. 10: Throughput in WiMAX for mobile node in integrated WiMAX - WLAN network

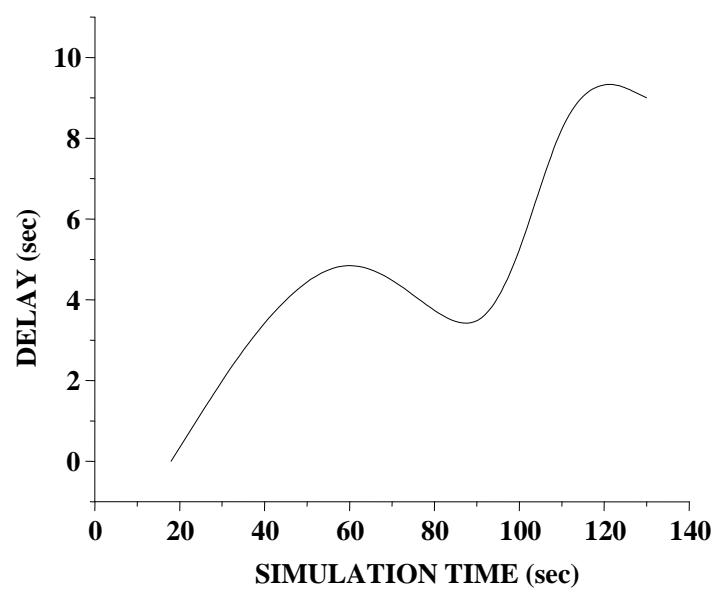

Fig. 11: Delay in WLAN for mobile node in integrated WiMAX - WLAN network

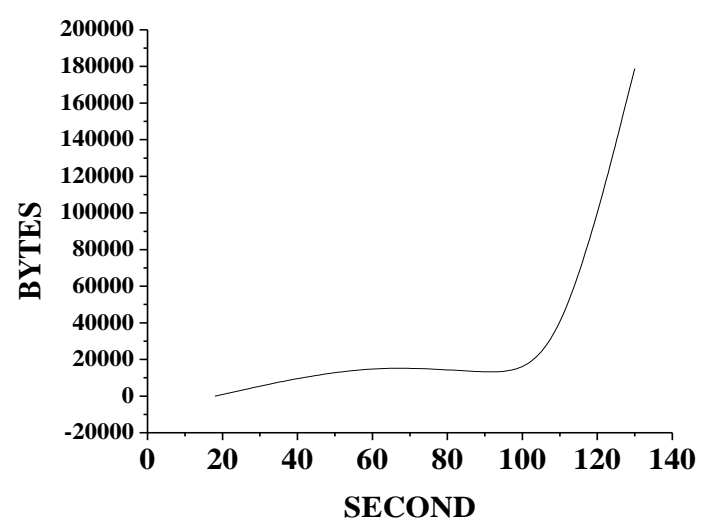

Fig. 12: Throughput in WLAN for mobile node in integrated WiMAX - WLAN network

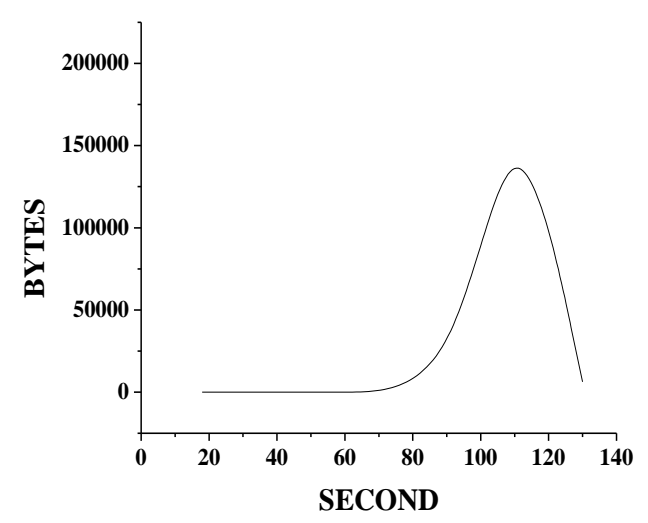

Fig. 13: Data Dropped in WLAN for mobile node in integrated WiMAX - WLAN network

\subsection{Discussion}

The simulations for mobile and fixed integrated WiMAXWLAN had been performed in rural environment with Outdoor to Indoor and Pedestrian path loss model under different network parameters. The Fig. 4 and Fig. 5 present the delay $\&$ throughput of WiMAX network during the fixed integration scheme. Fig. 6 to Fig. 8 depict the delay, throughput and data dropped for the WLAN network for fixed integration of $2 \mathrm{G}$ and $4 \mathrm{G}$ networks. The Fig. 9 and Fig. 10 present the delay and throughput of WiMAX network during the mobile integration scheme. Fig. 11 to Fig. 13 depicts the delay, throughput and data dropped respectively for the WLAN network for mobile integration of $2 \mathrm{G}$ and $4 \mathrm{G}$ networks. As the simulations move from fixed to mobile integration system of $2 \mathrm{G}-4 \mathrm{G}$ network, the degradations has been observed in the results of WLAN and WiMAX due the pervasive environment of mobility and path loss as presented in Fig. 9 to Fig. 13. The amount of delay and dropped data for WiMAX-WLAN had been increased in mobile integrated scheme as compared to the fixed integration scheme of $2 \mathrm{G}-4 \mathrm{G}$ networks as shown in Fig. 9, Fig. 11 and Fig. 13. The decrease in the throughput of the WiMAX in Fig. 10 and Fig. 12 for WLAN system can be observed in fixed integration scheme. 


\section{CONCLUSION AND FUTURE SCOPE}

The work in the present paper analyzed the performance of the integration schemes of WiMAX and WLAN networks under pervasive environment of mobility and path loss. It was observed from the performance analysis of WiMAX-WLAN network for fixed and mobile integration system that the fixed integration scheme worked well as compared to mobile integrated system. The performance of the mobile integrated system degraded due to combined effect of the mobility \& path loss. But both fixed and mobile integrated network worked well as compared to the single network, because it provided the satisfactory throughput to the end users. In future, the work can be extended to enhance the signal strength of integrated $2 \mathrm{G}-4 \mathrm{G}$ network.

\section{REFERENCES}

[1] Bartels S., Pearson M., "Wireless Local Area Network Planning: An Overview", New Zealand Computer Science Research Students' Conference, Vol., No. , 2008, pp: 188-191.

[2] Rappaport T. S., "Wireless Communication: Design, Principles and Practice", 2nd Ed., 2002, Prentice Hall.

[3] Jha R. K., Dalal U. D., "A Journey on WiMAX and its Security Issues", International Journal of Computer Science and Information Technologies, Vol. 1, No. 4, 2010, pp: 256-263

[4] A. Konak, "Estimating path loss in wireless local area network using ordinary kriging," in Proceeding of Winter Simulation Conference, 2010, pp. 2888 - 2896.

[5] Shrestha P. L., Hempel M. H., Mahasukhon P., Ma T., Sharif H., "A Performance comparison of mobile WiMAX spectrums: $2.5 \mathrm{GHz}$ vs. $3.65 \mathrm{GHz}$ ", in Proceeding of IEEE Vehicular Technology Conference, 2012, pp: 1-5.

[6] Ain K., Tarafder M. S. R., Khan S. A., Ali M. L., "Path loss compensation technique for WiMAX technology based communication system", International Journal of Engineering Science and Technology, Vol. 3 No. 9, 2011, pp: 7226-7233

[7] Zaballos A., Corral G., Carne A., Pijoan J. L., " Modelling a new propagation model for WLAN", www.salle.url.edu/zaballos/OPNET2004b.pdf.

[8] Mardeni R., T. Siva Priya "Optimised COST-231 Hata Models for WiMAX path loss prediction in suburban and open urban environments", Canadian Center of Science and Education, Vol. 4, No. 9, 2010, pp: 74-89.

[9] Priyanka, Malhotra J., "Performance evaluation of WiMAX networks through large scale fading channel conditions", International Journal of Computer Applications, Vol. 70, No. 2, 2013, pp: 22-25.

[10] Lin H. T., Lin Y. Y., Chang W. R., Cheng R. S., "An integrated WiMAX/Wi-Fi architecture with QoS consistency over broadband wireless networks", in Proceedings of IEEE Consumer Communications and Networking Conference, 2009, pp. 1-7.

[11] Benoubira S., Frikha M., Tabbane S., "Mobility and QoS management in heterogeneous wireless networks", International Journal of Computer Science and Network Security, Vol. 11, No. 9, 2011, pp:82-90.

[12] Rizvi S., Aziz A, Saad N. M., Armi N., Yusoff M. Z., "Tight Coupling internetworking between UMTS and WLAN: challenges, design architectures and simulation analysis" International Journal of Computer Networks, Vol. 3, No. 2, 2011,pp:116-134. 